Bull. Austral. Math. Soc.

Vol. 41 (1990) [435-439]

\title{
AREA AND LENGTH MAXIMA FOR UNIVALENT FUNCTIONS SHINJI YAMASHITA
}

Let $S$ be the family of functions $f(z)=z+a_{2} z^{2}+\ldots$ which are analytic and univalent in $|z|<1$. We find the value

$$
\max _{f \in S} \iint_{|z|<r}\left|(z / f(z))^{\prime}\right|^{2} d x d y
$$

as a function of $r, 0<r<1$. The known lower estimate of

$$
\sup _{f \in S} \int_{|z|=r}\left|f^{\prime}(z)\right||d z|
$$

is improved. Relations with the growth theorem are considered and the radius of univalence of $f(z) / z$ is discussed.

For $g$ analytic in $D=\{|z|<1\}$, we set

$$
\triangle(r, g)=\iint_{|z|<r}\left|g^{\prime}(z)\right|^{2} d x d y, \quad 0<r \leqslant 1, \quad z=x+i y .
$$

We call $g$ Dirichlet-finite if $\triangle(1, g)<\infty$. Let $S$ be the family of functions

$$
f(z)=z+\sum_{n=2}^{\infty} a_{n} z^{n}
$$

which are analytic and univalent in $D$ and set

$$
F_{f}(z)=f(z) / z, \quad z \in D, \quad f \in S .
$$

As a consequence of the celebrated de Branges theorem: $\left|a_{n}\right| \leqslant n(n \geqslant 2)$ for $f \in S$, (see [1]) we have immediately

$$
\pi^{-1} \triangle\left(r, F_{f}\right)=\sum_{n=1}^{\infty} n\left|a_{n+1}\right|^{2} r^{2 n} \leqslant \sum_{n=1}^{\infty} n(n+1)^{2} r^{2 n}=\pi^{-1} \triangle\left(r, F_{K}\right),
$$

where $K(z)=z /(1-z)^{2}$ is the Koebe function. Therefore

$$
\max _{f \in S} \Delta\left(r, F_{f}\right)=2 \pi r^{2}\left(r^{2}+2\right)\left(1-r^{2}\right)^{-4}
$$

for $0<r<1$. For each $r, 0<r<1$, the maximum is attained only by the rotations of the Koebe function: $K_{\theta}(z)=e^{-i \theta} K\left(e^{i \theta} z\right)$, where $\theta$ is real. We first prove:

Received 28 June 1989

Copyright Clearance Centre, Inc. Serial-fee code: 0004-9729/90 \$A2.00+0.00. 
ThEOREM 1. We have

$$
\max _{f \in S} \triangle\left(r, 1 / F_{f}\right)=2 \pi r^{2}\left(r^{2}+2\right) \quad \text { for } \quad 0<r \leqslant 1 .
$$

For each $r, 0<r \leqslant 1$, the maximum is attained only by $K_{\theta}$ 's.

Proof: Given $f \in S$, we can apply the area theorem $[3, \mathrm{p} .29]$ to

$$
f(1 / z)^{-1}=z-a_{2}+\sum_{n=1}^{\infty} b_{n} z^{-n} \quad(|z|>1)
$$

to obtain

$$
\sum_{n=1}^{\infty} n\left|b_{n}\right|^{2} \leqslant 1
$$

Since

$$
1 / F_{f}(z)=1-a_{2} z+\sum_{n=1}^{\infty} b_{n} z^{n+1}, \quad z \in D
$$

it follows from (2), together with $\left|a_{2}\right| \leqslant 2$, that

$$
\begin{aligned}
\pi^{-1} \triangle\left(r, 1 / F_{f}\right) & =\left|a_{2}\right|^{2} r^{2}+2 r^{4} \sum_{n=1}^{\infty} 2^{-1}(n+1)\left|b_{n}\right|^{2} r^{2 n-2} \\
& \leqslant 4 r^{2}+2 r^{4} \sum_{n=1}^{\infty} n\left|b_{n}\right|^{2} \leqslant 2 r^{2}\left(r^{2}+2\right) .
\end{aligned}
$$

Since $\Delta\left(r, 1 / F_{K_{\theta}}\right)=2 \pi r^{2}\left(r^{2}+2\right)$, we now have the identity. If the maximum is attained by $f$, then $\left|a_{2}\right|=2$, so that $f=K_{\theta}$ for some $\theta$.

It follows that $\Delta\left(1,1 / F_{f}\right) \leqslant 6 \pi$. This shows that each function $f \in S$ is the quotient of two functions, $z$ and $1 / F_{f}(z)$, both of which are bounded and Dirichletfinite in $D$; see estimate (6) for the bound $\left|1 / F_{f}\right| \leqslant 4$.

Each $f \in S$ maps $\{|z|=r\}$ onto a curve of length

$$
L(r, f)=r \int_{0}^{2 \pi}\left|f^{\prime}\left(r e^{i t}\right)\right| d t \quad(0<r<1) .
$$

It is known that, for $0<r<1$,

$$
2^{-1} \pi r(1+r)(1-r)^{-2}<L(r, K) \leqslant \sup _{f \in S} L(r, f) ;
$$

see [2, Theorem 2] and [3, p.39]. Now, as another application of the de Branges theorem we have

$$
\max _{f \in S} \Delta(r, f)=\Delta(r, K)=\pi r^{2}\left(r^{4}+4 r^{2}+1\right)\left(1-r^{2}\right)^{-4},
$$

for $0<r<1$. The maximum is attained only by $K_{\theta}$ 's.

We improve (3) in 
ThEOREM 2. For $0<r<1$ we have

$$
2 \pi r\left(r^{4}+4 r^{2}+1\right)^{1 / 2}\left(1-r^{2}\right)^{-2} \leqslant L(r, K) \leqslant \sup _{f \in S} L(r, f)
$$

Proof: This is a consequence of the expression of $\Delta(r, K)$ in (4), without appealing to the expression of $L(r, K)$ in terms of elliptic integrals (see [2]). We only apply to $K$ the isoperimetric inequality:

$$
\Delta(r, f) \leqslant \pi\{L(r, f) /(2 \pi)\}^{2} \text { for } f \in S
$$

which says that, of all rectifiable Jordan curves with the given perimeter $L(r, f)$, $(0<r<1)$, the circle has interior of maximum area.

Since

$$
\inf _{0<r<1}\left(r^{4}+4 r^{2}+1\right)^{1 / 2}(1+r)^{-3}=\sqrt{6} / 8>1 / 4,
$$

estimate (5) is better than (3).

We recall that

$$
L(r, f) \leqslant 2 \pi r(1-r)^{-2} \equiv \gamma(r)
$$

for $f \in S$ and $0<r<1[3$, p.40]. Estimate (5) now yields

$$
(\sqrt{6} / 4) \gamma(r) \leqslant \sup _{f \in S} L(r, f) \leqslant \gamma(r)
$$

Note that $\gamma(r)$ is the length of the boundary circle of $\delta_{r}=\left\{|z|<r(1-r)^{-2}\right\}$.

We recall the growth theorem for $f \in S$ :

$$
(1+|z|)^{-2} \leqslant\left|F_{f}(z)\right| \leqslant(1-|z|)^{-2}, \quad z \in D
$$

see [3, p.33]. The image $f(\{|z|<r\})(f \in S)$ is contained in the disc $\delta_{r}$ with area $\pi r^{2}(1-r)^{-4}$ and

$$
\Delta(r, f) /\left\{\pi r^{2}(1-r)^{-4}\right\}
$$

is at most:

$$
\left(r^{4}+4 r^{2}+1\right)(1+r)^{-4}, \quad 0<r<1
$$

which decreases from 1 to $3 / 8$ as $r$ increases from 0 to 1 . Therefore, one may say that the upper estimate of (6) becomes "worse" as $r$ increases because $f(\{|z|<r\})$ occupies only a small part of $\delta_{r}$ in area. We next assume that $F_{f}$ is nonconstant. The Riemann surface $\Phi_{r}$ ( $\Phi_{r}^{*}$, respectively) which is the image of $\{|z|<r\}$ by $F_{f}$ $\left(1 / F_{f}\right.$, respectively), by (6), has projection contained in the disc with centre 0 and 
radius $(1-r)^{-2}\left((1+r)^{2}\right.$, respectively $)$. The "sheet-number" of the covering surface $\Phi_{r}\left(\Phi_{r}^{*}\right.$, respectively) over this disc:

$$
\triangle\left(r, F_{f}\right) /\left\{\pi(1-r)^{-4}\right\}\left(\Delta\left(r, 1 / F_{f}\right) /\left\{\pi(1+r)^{4}\right\}, \text { respectively }\right)
$$

is at most $2 r^{2}\left(r^{2}+2\right)(1+r)^{-4}$ which increases from 0 to $3 / 8$ as $r$ increases from 0 to 1. In this sense (6) yields little information on the distribution of the values of $F_{f}(z)$ $\left(1 / F_{f}(z)\right.$, respectively), for $|z|<r$.

Let $C$ be the family of all $f \in S$ such that $f(D)$ is convex. With the aid of the coefficient estimate $[3, \mathrm{p} .45$, Corollary $]$ we have

$$
\max _{f \in C} \triangle\left(r, F_{f}\right)=\pi r^{2}(1-r)^{-2}, \quad 0<r<1 .
$$

For each $r, 0<r<1$, the maximum is attained only by $J_{\theta}(z) \equiv z /\left(1-e^{i \theta} z\right), z \in D$, $\theta$ real. A natural conjecture is that

$$
\max _{f \in C} \triangle\left(r, 1 / F_{f}\right)=\pi r^{2}, \quad 0<r \leqslant 1,
$$

where the maximum is attained only by $J_{\theta}$ 's.

REMARK. If $a_{2}=0$ in (1) for $f \in S$, then $F_{f}^{\prime}(0)=a_{2}=0$, so that $F_{f}$ is not univalent in any disc with centre 0 . To consider the case $a_{2} \neq 0$, we first note that the function

$$
\varphi(x)=-\log \left(1-x^{2}\right)+\left(3 x^{2}-2 x^{4}\right)\left(1-x^{2}\right)^{-2}
$$

increases from 0 to $+\infty$ as $x$ increases from 0 to 1 . Therefore there exists a number $R \equiv R\left(a_{2}\right), 0<R<1$, such that $\varphi(R)=\left|a_{2}\right|^{2}$. We shall show that $F_{f}$ is univalent in $\left\{|z|<R\left(a_{2}\right)\right\}$. The expression for $1 / F_{f}$ in the proof of Theorem 1 shows that

$$
g(z) \equiv\left\{1-1 / F_{f}(z)\right\} / a_{2}=z-a_{2}^{-1} \sum_{n=2}^{\infty} b_{n-1} z^{n} \text { in } D .
$$

The Schwarz inequality, together with (2), yields that

$$
\begin{gathered}
\left|a_{2}\right|^{-1} \sum_{n=2}^{\infty} n\left|b_{n-1}\right| R^{n-1} \\
\leqslant\left|a_{2}\right|^{-1}\left\{\sum_{n=2}^{\infty}(n-1)\left|b_{n-1}\right|^{2}\right\}^{1 / 2}\left\{\sum_{n=2}^{\infty} n^{2}(n-1)^{-1}\left(R^{2}\right)^{n-1}\right\}^{1 / 2} \\
\leqslant\left|a_{2}\right|^{-1} \varphi(R)^{1 / 2}=1 .
\end{gathered}
$$


By [3, p.73, Problem 24 (b)] we have that $R^{-1} g(R z)$ is univalent and starlike in $D$. Thus $g$, and hence $F_{f}$, are univalent in $\{|z|<R\}$ as we wished. We note that the image of $\{|z|<R\}$ under $1 / F_{f}$ is starlike with respect to 1 also. Unfortunately we cannot claim that $R\left(a_{2}\right)$ is sharp. In fact, for the Koebe function $K$ with $a_{2}=2$ we have

$$
R(2)=0.6823 \ldots \ldots,
$$

while $F_{K}$ is univalent in $D$. Finally, since $\left|a_{2}\right| \leqslant 2$ for $f \in S$, we have $R\left(a_{2}\right) \leqslant R(2)$.

\section{REFERENCES}

[1] L. de Branges, 'A proof of the Bieberbach conjecture', Acta Math. 154 (1985), 137-152.

[2] P.L. Duren, 'An arclength problem for close-to-convex functions', J. London Math. Soc. 39 (1964), 757-761.

[3] P.L. Duren, Univalent functions (Springer-Verlag, New York, Berlin, Heidelberg, Tokyo, 1983).

Department of Mathematics

Tolyo Metropolitan University

Fukasawa, Setagaya, Tokyo 158

Japan 